\title{
Green fabrication route of robust, biodegradable silk sericin and poly(vinyl alcohol)
}

\section{nanofibrous scaffolds}

Pongsathorn Kumkun ${ }^{1}$, Nantaprapa Tuancharoensri ${ }^{2}$, Gareth Ross ${ }^{1,2}$, Sararat Mahasaranon ${ }^{1}$, Jirapas Jongjitwimol $^{3}$, Paul D. Topham ${ }^{4}$, Sukunya Ross ${ }^{1,2, *}$

${ }^{1}$ Program in Industrial Chemistry, Biopolymer Group, Department of Chemistry, Faculty of Science, Naresuan University, Phitsanulok, Thailand 65000

${ }^{2}$ Excellent Center of Biomaterials, Department of Chemistry, Faculty of Science, Naresuan University, Phitsanulok 65000, Thailand

${ }^{3}$ Clinical Microbiology, Department of Medical Technology, Faculty of Allied Health Sciences, Naresuan University, Phitsanulok, Thailand 65000

${ }^{4}$ Aston Institute of Materials Research, Aston University, Birmingham, B4 7ET, UK

* Corresponding author at: Ross S. Biopolymer Group, Department of Chemistry, Faculty of Science, Naresuan University, Phitsanulok, Thailand 65000. Tel.: +66 55 963445: fax: +66 55 963402. Email address: sukunyaj@nu.ac.th

Keywords: silk sericin, poly(vinyl alcohol), electrospinning, nanofibers, scaffold, biodegradable

\begin{abstract}
Silk sericin (SS) has been extensively used to fabricate scaffolds for tissue engineering. However, due to its inferior mechanical properties, it has been found to be a poor choice of material when being electrospun into nanofibrous scaffolds. Here, SS has been combined with poly(vinyl alcohol) (PVA) and electrospun to create scaffolds with enhanced physical properties. Crucially, these SS/PVA nanofibrous scaffolds were created using only distilled water as a solvent with no added crosslinker in an environmentally friendly process. Temperature has been shown to have a marked effect on the formation of the SS sol-gel transition and thus influence the final formation of fibers. Heating the spinning solutions to $70{ }^{\circ} \mathrm{C}$ delivered nanofibers with enhanced morphology, water stability, and mechanical properties. This is due to the transition of SS from $\beta$-sheets into random coils that enables enhanced molecular interactions between SS and PVA. The most applicable SS/PVA weight ratios for the formation of nanofibers with the desired properties were found to be 7.5/1.5 and 10.0/1.5. The fibers had diameters ranging from 60 to $500 \mathrm{~nm}$, where higher PVA and SS concentrations promoted larger diameters. The crystallinity within the fibers could be controlled by manipulation of the balance between PVA and SS loadings. In vitro degradation (in phosphate buffer solution, $\mathrm{pH} 7.4$ at $37^{\circ} \mathrm{C}$ )
\end{abstract}


was 30-50\% within 42 days and fibers were shown to be non-toxic to skin fibroblast cells. This work demonstrates a new green route for incorporating SS into nanofibrous fabrics, with potential use in biomedical applications.

\section{Introduction}

Natural polymers, such as collagen, gelatin, chitin, chitosan, cellulose, alginate, silk fibroin and silk sericin, have been employed by many researchers for use in biomedical and cosmetic applications due to their biocompatibility and non-toxicity to the human body [1-12]. Silk sericin (SS) has recently gained interest among the range of natural polymers and has previously been used for the production of skin scaffolds $[13,14]$. SS is synthesized in the labial gland of the silkworm, Bombyx mori, that forms three layers around double strands of fibroin filaments in the silk thread [15]. It is a glycoprotein and comprises 18 different amino acids (mostly serine, glycine, aspartic acid and tyrosine, see chemical structures of SS in Fig. 2a) with strong polar side groups (hydroxyl, carboxyl and amino groups), which renders sericin water soluble and allows it to interact with other materials $[4,11]$. Structurally, SS consists of random coils and $\beta$-sheets where the sol-gel transition occurs in response to temperature, moisture absorption and mechanical stretching (tensile strength) [15].

Due to the physicochemical properties described, SS is a potential biocompatible material and has many desirable properties for biomedical applications, such as resistance to oxidation, antibacterial, biodegradable, anti-coagulation and anti-cancer [16-21]. SS-containing materials have been shown to take less time to heal compared to non-treated wounds [22,23]. However, due to its weak mechanical properties, SS generally needs to be processed with other biocompatible materials, such as chitosan [4], polyacrylamide [24] and poly(vinyl alcohol) (PVA) [13]. Notably, PVA has been widely used in medical applications due to its excellent physical properties and low cost, in addition to being biodegradable and biocompatible [12,25-27].

There are many factors that affect the physical and mechanical properties of SS-based scaffolds, such as molecular weight, temperature and component concentration [16,28-35]. For 
example, Um et al. [28] studied the effect of molecular weight on the structure and mechanical properties of silk sericin fabricated in various forms of gels, films and sponges. They found that increasing the molecular weight of SS causes an increase in gelation propensity of the SS aqueous solution, gel strength, gel-sol transition temperature, crystallinity and mechanical properties, but reduces porosity, swelling ratio and sponge density. Interestingly, the molecular weight changes of SS in their study did not have an effect on cell toxicity. Our previous work [13] has shown that the storage temperature after SS degumming has a significant effect on the secondary conformational structure of SS, which is mostly random coils or $\beta$-sheets when they were prepared at $25^{\circ} \mathrm{C}$ and $-10{ }^{\circ} \mathrm{C}$, respectively. In addition, it was found that porous PVA/SS (random coils) scaffolds with pore diameters between 20-30 $\mu \mathrm{m}$ showed efficiency for skin cell adhesion and proliferation.

One of the most popular processes to fabricate tissue engineering scaffolds is electrospinning, which produces large surface area and porous structure nanofibers that can be designed to be similar to the extracellular matrix (ECM). This enables the enhancement of cell adhesion, proliferation and migration [3,36-38]. Generally, SS has poor spinnability unless it is combined with another highly spinnable polymer [3,11,21,29]. During fabrication, water and acetic acid have been used as solvents, with and without crosslinker (i.e. glutaraldehyde) at room temperature. Montazer et al. [3] electrospun chitosan (Ch, $3 \% \mathrm{w} / \mathrm{v}) / \mathrm{sericin}$ (SS, $10 \% \mathrm{w} / \mathrm{v}) / \mathrm{PVA}$ $(10 \% \mathrm{w} / \mathrm{v})$ at different ratios using dilute acetic acid [3] to produce small diameter bead-less nanofibers with narrow size distribution. Yao et al. [11] electrospan PVA/SS solutions in deionized water at room temperature and found that the resulting PVA and PVA/SS fibers must be crosslinked (using glutaraldehyde) to prevent them from dissolving instantly in water.

In the work herein, we introduce a new green route for the production of SS-incorporated nanofibrous fabrics. Accordingly, we have studied the effect of spinning temperature on the physical and mechanical properties of electrospun SS/PVA nanofibers and have successfully fabricated robust nanofibers by heating spinning solutions at $70{ }^{\circ} \mathrm{C}$ using only distilled water, 
without crosslinker, for the first time. Crucially, heating the spinning solutions delivered nanofibers with better morphology, greater water stability and stronger mechanical properties, due to the transition of SS from $\beta$-sheets into random coils. This enables enhanced molecular interactions between PVA and SS, which subsequently equips the PVA/SS nanofibers with more water stability without using crosslinker. As concentration was found to be the most important parameter among the major factors in controlling the morphology and average fiber diameter [39], the effect of SS and PVA concentrations on the nanofiber properties (morphology, molecular interaction, crystallinity, degradation, and in vitro cell cytotoxicity) have been studied.

\section{Material and Method}

\subsection{Materials}

Silk cocoons (Bombyx mori) were provided from Tak province in the lower northern region of Thailand. Poly(vinyl alcohol) (PVA, $M_{w} \sim 80,000$ Da and $\sim 120,000 \mathrm{Da}$ ) were supplied by SigmaAldrich Co. Inc, Singapore. For cytotoxicity, 2,3-bis-(2-methoxy-4-nitro-5-sulfophenyl)-2Htetrazolium-5-carboxanilide salt (XTT, from Sigma-Aldrich, St. Louis, MO, USA) and normal human dermal fibroblast (NHDF) cell line (from American Type Culture Collection, USA) were provided by Center of Excellence for Innovation in Chemistry, Department of Pharmaceutical Technology, Faculty of Pharmaceutical Science, Naresuan University.

\subsection{Preparation of silk sericin powders}

Silk sericin (SS) was prepared from a simple hot-water degumming process following our previously employed method [13], which gave a high yield of $23 \mathrm{wt} \%$ SS powders. Briefly, $20 \mathrm{~g}$ of silk cocoons were weighed and cut into small pieces and then added to $500 \mathrm{~mL}$ of DI water before heating at 100

${ }^{\circ} \mathrm{C}$ for 4 hours. After the extraction process, the sericin solution was stored at room temperature (25 ${ }^{\circ} \mathrm{C}$ ) for 24 hours before drying into the final powder in an oven at $60{ }^{\circ} \mathrm{C}$ and stored in desiccator before use.

\subsection{Preparation of PVA/SS blend solutions}


Both PVA and SS solutions were prepared separately before mixing at the appropriate composition shown in Table 1 (for study the effect of spinning temperature in Section 3.1) and Table 2 (for all heated solutions to study the effect of SS and PVA concentration in Section 3.2). A PVA stock solution was prepared by dissolving PVA pellets in distilled water at $110^{\circ} \mathrm{C}$ for 2 hours in a closed system. The SS solutions were prepared by dissolving SS powder (from Section 2.2) in distilled water at 70 ${ }^{\circ} \mathrm{C}$ for 2 hours, where a fresh solution was prepared for every sample. PVA and SS solutions at the desired compositions were mixed and heated at $70{ }^{\circ} \mathrm{C}$ for 30 mins under reflux in order to make homogeneous solutions.

Table 1. Compositions of non-heated and heated SS/PVA solutions (distilled water) to study the effect of temperature on the electrospinning process.

\begin{tabular}{lcc}
\hline Sample Name & SS $(\% \mathrm{w} / \mathrm{v})$ & PVA $(\% \mathrm{w} / \mathrm{v})$ \\
\hline Non-heated SS/PVA & 1.5 & 7.5 \\
Heated SS/PVA-1 & 1.5 & 7.5 \\
Heated SS/PVA-2 & 1.5 & 10.0 \\
\hline
\end{tabular}

Table 2. Compositions of heated SS/PVA solutions (distilled water) to study the effect of SS and PVA concentration during electrospinning.

\begin{tabular}{cccccc}
\hline Sample code & $\begin{array}{c}\text { SS } \\
(\% \mathrm{w} / \mathrm{v})\end{array}$ & $\begin{array}{c}\text { PVA } \\
(\% \mathrm{w} / \mathrm{v})\end{array}$ & Sample code & $\begin{array}{c}\text { SS } \\
(\% \mathrm{w} / \mathrm{v})\end{array}$ & $\begin{array}{c}\text { PVA } \\
(\% \mathrm{w} / \mathrm{v})\end{array}$ \\
\hline PVA & - & $5.0,7.5,10.0$ & EP5 & 1.5 & 7.5 \\
EP1 & 0.75 & 5.0 & EP6 & 3.0 & 7.5 \\
EP2 & 1.5 & 5.0 & EP7 & 0.75 & 10.0 \\
EP3 & 3.0 & 5.0 & EP8 & 1.5 & 10.0 \\
EP4 & 0.75 & 7.5 & EP9 & 3.0 & 10.0 \\
\hline
\end{tabular}

\subsection{Fabrication of SS/PVA nanofibers}

Mixtures of SS and two different molecular weight PVA samples $\left(M_{w} \sim 80,000 \mathrm{Da}\right.$ and $M_{w} \sim 120,000$ Da) in distilled water (without any other plasticizers and/or chemical solvents) were loaded into a $10 \mathrm{~mL}$ syringe with $22 \mathrm{G} \times 1 \frac{1}{2}$ inch needle ( $0.7 \times 40 \mathrm{~mm}$ thin wall hypodermic needle). The syringe 
containing sample solution was sheathed in a silicone heating mat, which was connected to a heating control unit, see Fig. 2 b, allowing the sample solution to be held to $70{ }^{\circ} \mathrm{C}$ during the electrospinning process. The electrospinning process parameters were studied to identify the optimum parameters to be used: feeding rate $1.2 \mathrm{~mL} /$ hour, distance from needle to collector of $15 \mathrm{~cm}$ and $25 \mathrm{kV}$ voltage. Of note, the SS/PVA (PVA $M_{w} \sim 120,000 \mathrm{Da}$ ) solution did not form fibers during spinning due to too the high viscosity of PVA while SS/PVA (PVA $M_{w} \sim 80,000 \mathrm{Da}$ ) showed good formation under the same operating conditions. Therefore, PVA $\left(M_{w} \sim 80,000 \mathrm{Da}\right)$ was selected for further study, as described herein.

\subsection{Characterization}

\section{Morphology}

Morphology of the PVA/SS electrospun nanofibers was observed using scanning electron microscopy (SEM) (Leo model 1455VP). Samples were cut into small pieces before being mounted onto metal stubs and dried overnight prior to gold deposition to enhance the electrical conductivity. Different areas of the sample surface were investigated.

\section{Conformational structures and intermolecular bonding study}

The conformational structure of silk sericin before and after electrospinning were assessed using Attenuated Total Reflection-Fourier Transform Infrared (ATR-FTIR, PerkinElmer Spectrum GX, 4000- $400 \mathrm{~cm}^{-1}$ ) spectroscopy. The intermolecular bonding of SS and PVA in the temperature controlled electrospun nanofibers with different concentrations of SS and PVA were also observed by ATR-FTIR.

\section{Tensile properties}

The tensile properties of the obtained fiber mats (with dimensions: length $=40 \mathrm{~mm}$, width $=25 \mathrm{~mm}$ and thickness $=0.40 \mathrm{~mm})$ were measured by a universal testing machine $\left(\right.$ model 5965 INSTRON ${ }^{\circ}$ CALIBRATION LAB) at a crosshead speed of $0.06 \mathrm{~mm} / \mathrm{min}$ with load cell of $100 \mathrm{~N}$. The samples were tested in triplicate.

\section{Crystallinity}


The crystallinity of all samples was investigated by X-ray diffraction (XRD, Philips model X'Pert Pro). Diffraction patterns were recorded with a diffraction angle $(2 \theta)$ in the range of $5^{\circ}$ to $50^{\circ}(\mathrm{Cu}$ $\mathrm{K} \alpha, \lambda=1.54 \AA)$.

\section{Contact angle}

Hydrophilicity of the electrospun fibers was observed by water contact angle (CA, Dataphysics Model OCA20) at room temperature. Water was injected from a syringe onto the surface of the nanofiber mats at $2.0 \mu \mathrm{L}$ dosing volume. Images of the water drop were recorded by video camera. The contact angles of samples were analyzed using the built in SCA software.

\section{Thermal property of SS powders}

The thermal properties of the SS powders were investigated using Differential Scanning Calorimeter (DSC, Mettler model DAC1). Samples were heated from 25 to $250{ }^{\circ} \mathrm{C}$, at a heating rate of $10{ }^{\circ} \mathrm{C}$ $\min ^{-1}$ under a nitrogen atmosphere.

\section{Degradation test}

Nanofiber mats were cut into $1 \mathrm{~cm} \times 1 \mathrm{~cm}$ with controlled thicknesses and then soaked in phosphate buffer solution ( $\mathrm{pH}$ 7.4) before being placed into the incubator at a controlled temperature of $37^{\circ} \mathrm{C}$. The weight loss of samples was measured at 1, 7, 14, 21, 28, 35 and 42 days with each sample tested in triplicate.

\subsection{In vitro cytotoxicity}

The cytotoxicity of the SS/PVA electrospun nanofibers was examined by the XTT (2,3-bis-(2methoxy-4-nitro-5-sulfophenyl)-2H-tetrazolium-5-carboxanilide salt) assay in normal human dermal fibroblast (NHDF) cell line. The fiber mats $\left(1 \mathrm{x} 1 \mathrm{~cm}^{2}\right)$ were impregnated in $1 \mathrm{~mL}$ of DMEM medium with serum free at room temperature for 24 hours. NHDF cell line suspensions were placed in 96-well plates at a density $1 \times 10^{4}$ cells/well and incubated in DMEM containing $10 \%$ fetal bovine serum (FBS), penicillin, streptomycin and amphotericin $\mathrm{B}$, at $37{ }^{\circ} \mathrm{C}$ under $\mathrm{CO}_{2}$ atmosphere for 24 hours. The medium was discarded and washed with PBS. Cells were then treated with both of sterilizing impregnated medium of nanofibers (EP5 and EP8) in each well before being incubated at 
$37{ }^{\circ} \mathrm{C}$ under $\mathrm{CO}_{2}$ for 24 hours. After treatment, the cells were washed with PBS and replaced with $200 \mu \mathrm{L}$ of the new serum-free medium before adding $50 \mu \mathrm{L}$ of XTT solution and incubated for a further 4 hours. The absorbance of the solution at $490 \mathrm{~nm}$ was measured by a microplate reader. Cell viability was calculated based on the following equation:

$$
\text { Cell viability }(\%)=\frac{O D_{s}}{O D_{c}} \times 100
$$

Where $\mathrm{OD}_{\mathrm{s}}$ is the absorbance of impregnated medium of nanofibers and $\mathrm{OD}_{\mathrm{c}}$ is the absorbance of a control (incubated with culture media without nanofibers or untreated cells). All experiments were performed three times with three replicate wells for every sample and control per assay. The optical images of the NHDF cell line were also observed after testing.

\section{Results and discussion}

\subsection{The exploration of the technique for fabrication of SS/PVA electrospun nanofibers: Effect of spinning temperature}

From our previous work [13], we found that the conformational structure of SS, which is temperature dependent, affects the formation of porous crosslinked PVA/SS scaffolds, in which random coil structures help to promote interactions between PVA and SS more than $\beta$-sheet structures. In this work, therefore, the thermal behavior of SS powders was first observed by DSC, as shown in Fig. 1a. Two broad endothermic peaks were seen at $\sim 110{ }^{\circ} \mathrm{C}$ and $225^{\circ} \mathrm{C}$ due to the evaporation of water and thermal decomposition of SS, respectively $[16,40]$. Interestingly, a small phase transition can be observed between $40-75^{\circ} \mathrm{C}$, which is attributed to the conformational change of silk protein from $\beta$-sheets to random coils. In addition, it was found that SS powders are completely dissolved in water at $70{ }^{\circ} \mathrm{C}$. Generally, the solubility of SS in water increases when the sericin molecular conformation transforms from $\beta$-sheets to random coils [41]. 


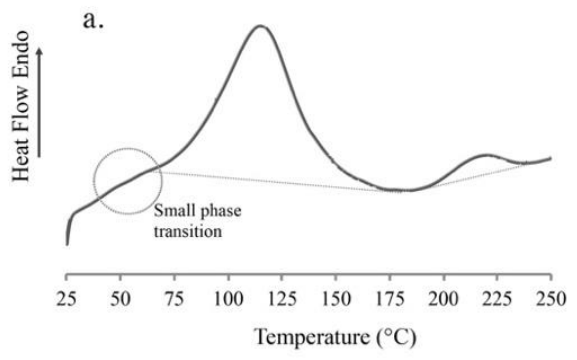

c.

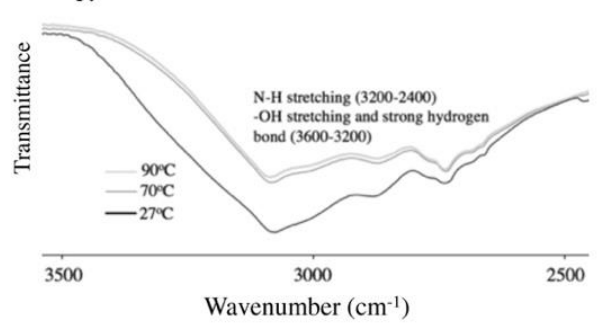

b.

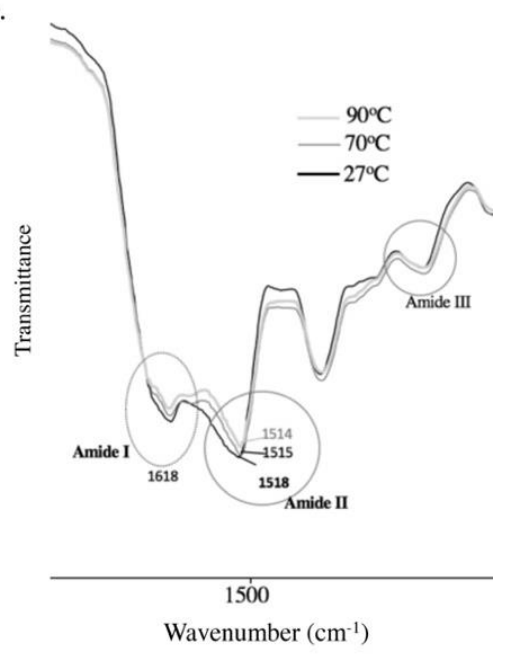

Figure 1. DSC thermogram of silk sericin powders (a) and FT-IR spectra of silk sericin solutions in two different regions to show the amide bands (b) and hydrogen bonding (c).

As silk sericin (SS) is comprised of amino acids, it contains amide I, II and III groups, which are involved in the secondary structures in proteins (random coils or $\beta$-sheet structures, see Fig. 2a) and can be determined using FT-IR spectroscopy [41-44]. In this work, SS conformational structures were studied by FT-IR at different temperatures from room temperature to $90^{\circ} \mathrm{C}$. The FT-IR spectra are shown in Fig. $1 \mathrm{~b}$ at $27{ }^{\circ} \mathrm{C}, 70{ }^{\circ} \mathrm{C}$ (temperature used for dissolving SS powders in water) and 90 ${ }^{\circ} \mathrm{C}$. The major bands of amide I (1670-1610 $\left.\mathrm{cm}^{-1}\right)$, II (1570-1515 $\left.\mathrm{cm}^{-1}\right)$ and III $\left(1260-1230 \mathrm{~cm}^{-1}\right)$ are attributed to the $\mathrm{C}=\mathrm{O}$ stretching vibrations of the amide groups, $\mathrm{N}-\mathrm{H}$ bending and in-phase combination of $\mathrm{N}-\mathrm{H}$ in plane bending and $\mathrm{C}-\mathrm{N}$ stretching vibrations, respectively [13]. The frequency bands of amide I, II and III (Fig. 1b) and the frequency band of N-H stretching (3200-2400 cm-1), $\mathrm{OH}$ stretching and strong hydrogen bonding $\left(3600-3200 \mathrm{~cm}^{-1}\right)$ of the SS solution at $27^{\circ} \mathrm{C}$ are broader than those seen at $70{ }^{\circ} \mathrm{C}$ and $90{ }^{\circ} \mathrm{C}$ (Fig. 1c). This is due to the high degree of hydrogen bonding in the $\beta$-sheet structures of the SS solution at lower temperatures [13]. SS has been shown to exhibit a reversible gel-sol transition somewhere between 70 and $90{ }^{\circ} \mathrm{C}$ (with the exact value being dependent on molecular weight [28]) due to the presence of breakable hydrogen bonds between the amino acid molecules. Accordingly, this behavior can be exploited to switch between an SS gel and sol by 
oscillating above and below the phase transition temperature [28,45-48].

Therefore, the electrospinning of SS and PVA in water with and without heating at $70{ }^{\circ} \mathrm{C}$ (samples named 'heated SS/PVA-1' and 'non-heated SS/PVA', respectively) was studied. The experimental set-up with temperature-controlling unit is shown in Fig. $2 b$, together with optical images of electrospun mats of both samples. Fig. 2c shows SEM images of heated SS/PVA-1 and non-heated SS/PVA and the cartoon schematic of the arrangement of PVA and SS chains after spinning at $70{ }^{\circ} \mathrm{C}$ and $27^{\circ} \mathrm{C}$ (room temperature, $\mathrm{T}_{\mathrm{r}}$ ). It was found that non-heated SS/PVA solutions produced fibrous fabrics with a greater number of beads and microdroplets (back circles) than heated SS/PVA-1 solutions. The fibrous mats produced from heated solutions were also mechanically stronger and tougher (see Fig. 3a), which is attributed to stronger molecular interaction between PVA and SS at elevated temperature that is made possible by the more open, flexible SS molecular conformation. Conversely, the $\beta$-sheet crystal structure of SS at $27{ }^{\circ} \mathrm{C}$ causes the gelation of SS and separation from PVA chains, which inhibits the production of homogeneous and smooth electrospun fibers (seen as microdroplets).

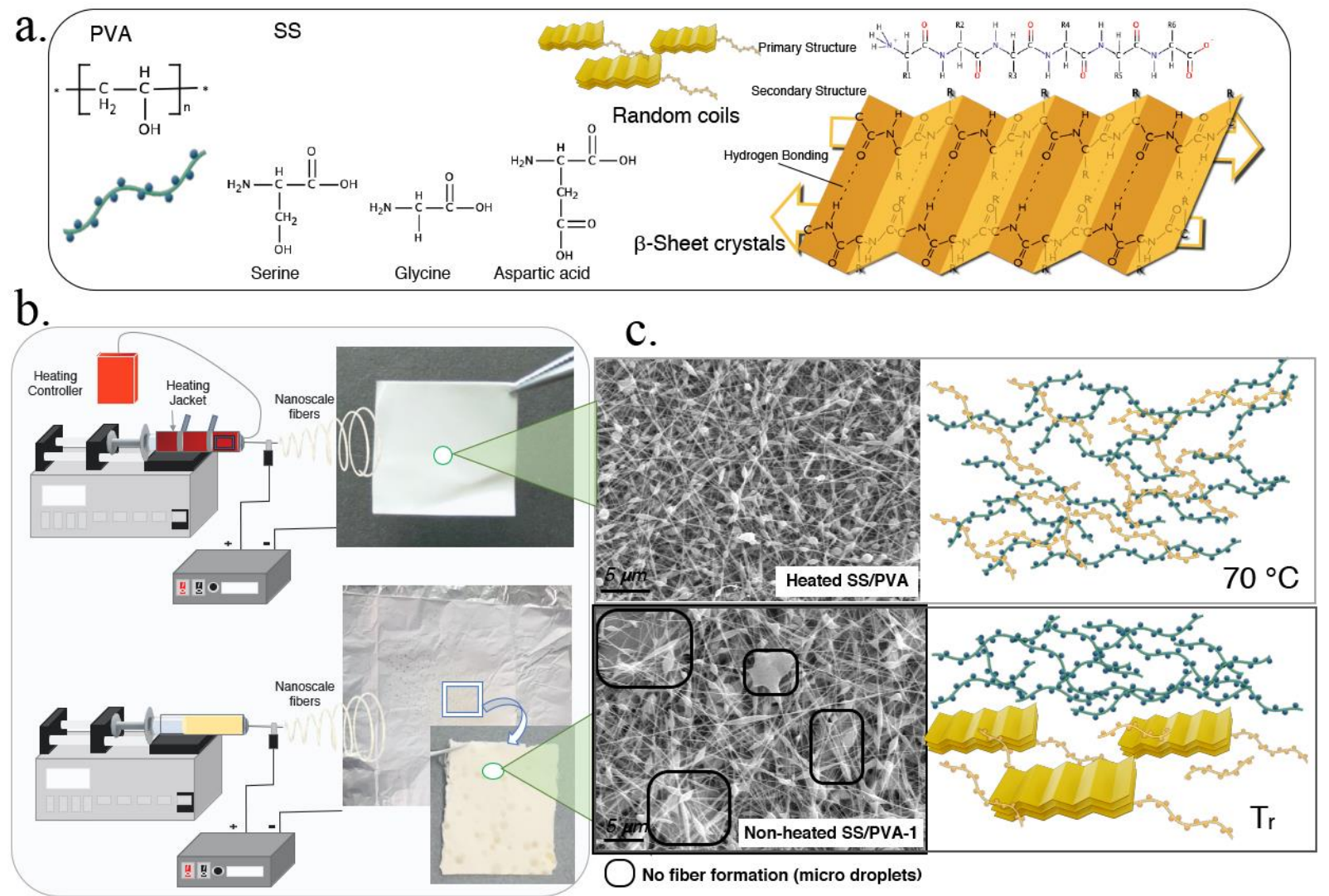


Figure 2. Schematic diagram of (a) the chemical and molecular structures of PVA and SS and (b) electrospinning set-up with and without heating and their optical electrospun mats and (c) SEM images of non-heated SS/PVA and heated SS/PVA-1 nanofibers with cartoons of their predicted molecular arrangement.
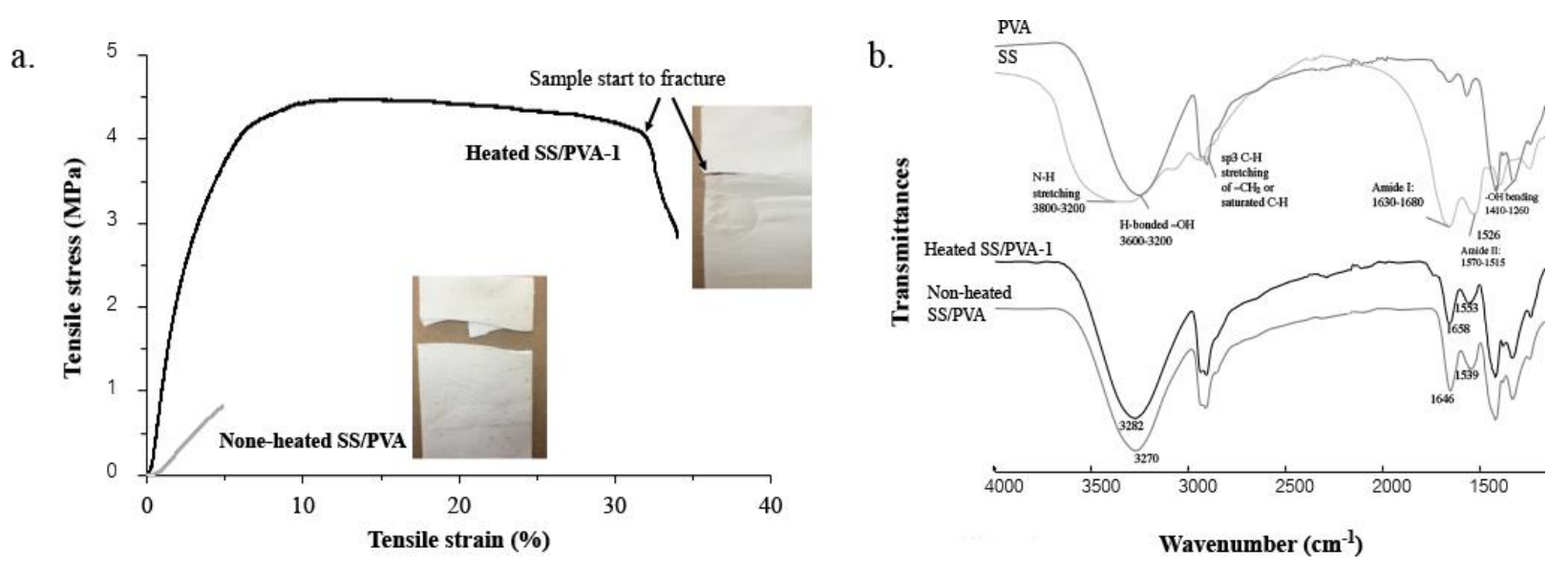

Figure 3. Stress-strain curves together with the samples after fracture (a) and FT-IR spectra (b) of non-heated SS/PVA and heated SS/PVA-1 nanofibers.

Fig. 3a shows that the tensile strength and \% elongation of heated SS/PVA-1 electrospun fibrous mats are substantially higher than those of non-heated mats. The heated SS/PVA-1 fiber mats started to fracture at $3.7 \mathrm{MPa}$ and then dropped to $2.8 \mathrm{MPa}$ before finishing the test without being completely fractured at $35 \%$ elongation. Conversely, the non-heated SS/PVA fiber mat fractures after applying the $0.7 \mathrm{MPa}$ stress with $5 \%$ elongation (also see supporting information for movies of the mechanical performance of each fiber mats). Fig. $3 b$ shows that the amide I and II bands of non-heated PVA/SS fabrics at 1646 and $1539 \mathrm{~cm}^{-1}$, respectively, were shifted to lower wavenumbers when compared to heated SS/PVA-1 mats $\left(1658 \mathrm{~cm}^{-1}\right.$ for amide I and 1553 $\mathrm{cm}^{-1}$ for amide II). This is due to the formation of hydrogen bonding between $\mathrm{C}=\mathrm{O}$ and $\mathrm{N}-\mathrm{H}$ groups in SS leading to the formation of $\beta$-sheet structures in non-heated solutions (and the resultant fibers). The frequency of $\mathrm{N}-\mathrm{H}$ stretching, and hydrogen bonded $-\mathrm{OH}$ bands of nonheated SS/PVA also appear at a lower wavenumber $\left(3270 \mathrm{~cm}^{-1}\right)$ than those of heated SS/PVA-1 samples $\left(3282 \mathrm{~cm}^{-1}\right)$. The high degree of hydrogen bonding between $\mathrm{C}=\mathrm{O}$ and $\mathrm{N}-\mathrm{H}$ groups in $\mathrm{SS}$ 
generates broad bands at room temperature.

Three samples of non-heated and heated SS/PVA electrospun fibers (Table 1) were submerged into PBS (pH 7.4) and left for 1 day before SEM was used to study their morphology (see Fig. 4). The images show that the non-heated fibers lost their fibrous structure completely after the first day in PBS, while heated fibers (SS/PVA-1 and SS/PVA-2) remained in good fibrous form, although heated SS/PVA-1 showed some signs of degradation. The water contact angle measurements of all samples are also shown in Fig. 4. Non-heated SS/PVA shows the lowest contact angle when compared to heated SS/PVA-1 and heated SS/PVA-2 nanofibers. The more hydrophobic surfaces in heated samples are a consequence of the enhanced interactions between the hydroxyl groups of PVA with SS, which reduces the amount of hydrophilic groups that are available on the surface of the fibers. In short, heating SS/PVA solutions at $70{ }^{\circ} \mathrm{C}$ during electrospinning promotes the formation of more hydrophobic fibrous fabrics, with stronger molecular interaction between PVA and SS occurring within the fibers, which duly enhances the mechanical properties of the fibers.

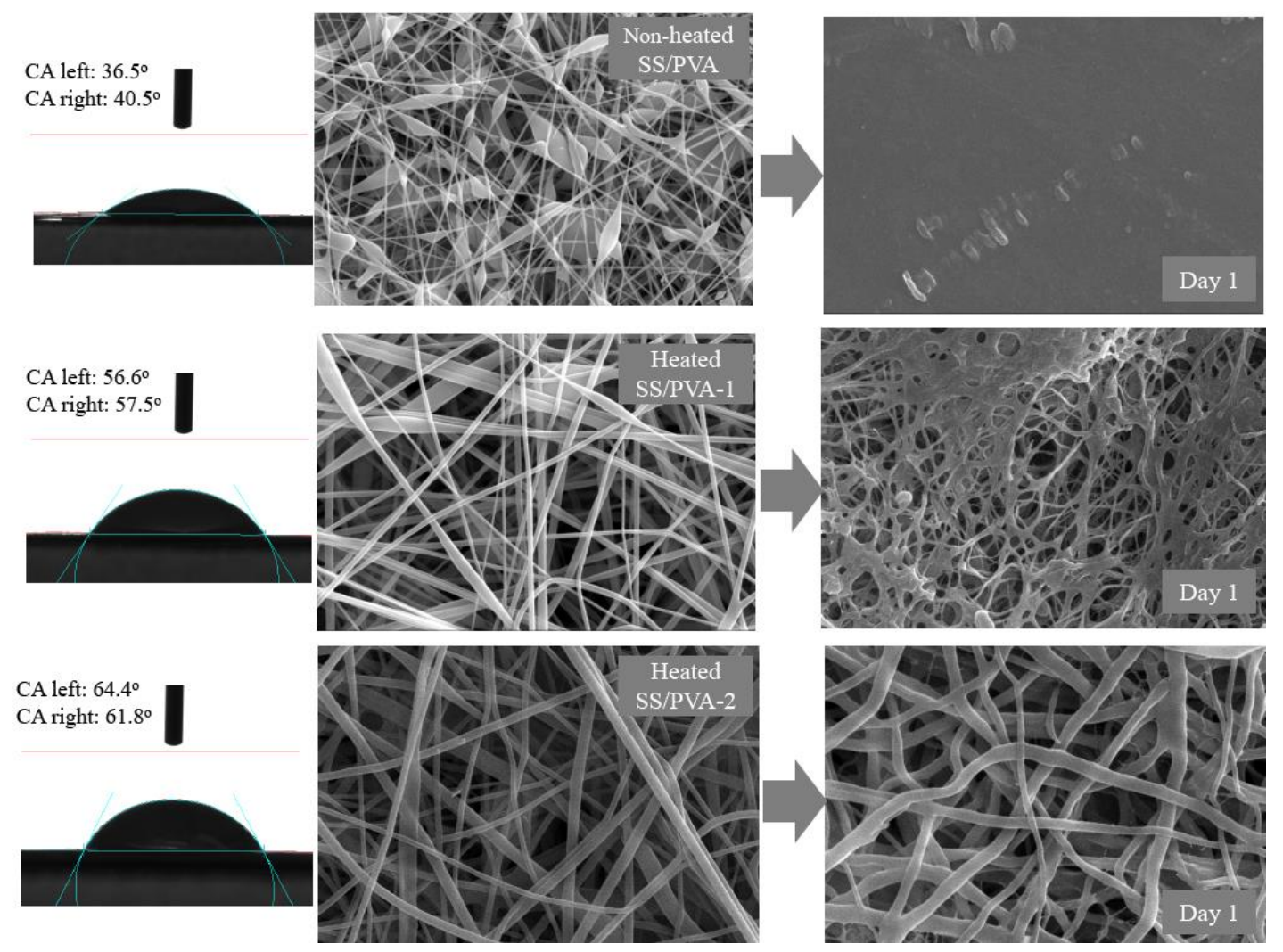

Figure 4. Water contact angle and SEM images of non-heated SS/PVA, heated SS/PVA-1 and heated SS/PVA-2 nanofibers after electrospinning and their SEM images after submersion in PBS for 1 day. 
The crystallinity of non-heated SS/PVA and heated SS/PVA-1 nanofibers, as well as neat SS and PVA nanofibers was investigated by XRD (see Fig. 5). Neat SS shows a broad peak representing an amorphous morphology, while neat PVA nanofibers show broad amorphous peaks alongside a small crystalline peak with low intensity at approximately $19^{\circ}$. Electrospinning is a quench process for processing polymers into fibers, where the solvent evaporates rapidly and effectively vitrifies the polymer chains far from equilibrium with a more amorphous structure than would be predicted based on thermodynamic principles [36]. However, heated and non-heated electrospun nanofibers show higher intensity crystalline peaks of PVA than neat PVA fibers. This suggests that SS promotes the alignment of the PVA chains, perhaps acting as a weak nucleating agent. In addition, non-heated SS/PVA mats show the highest amount of PVA crystallinity because the formation of non-fibrous features (as seen from SEM image in Fig. 4) is more prevalent during the electrospinning process. Non-fibrous features tend to be formed when the solvent molecules become trapped and therefore the solvent evaporates slower (than conventional fiber formation in electrospinning), which provides more mobility for chain alignment to occur.

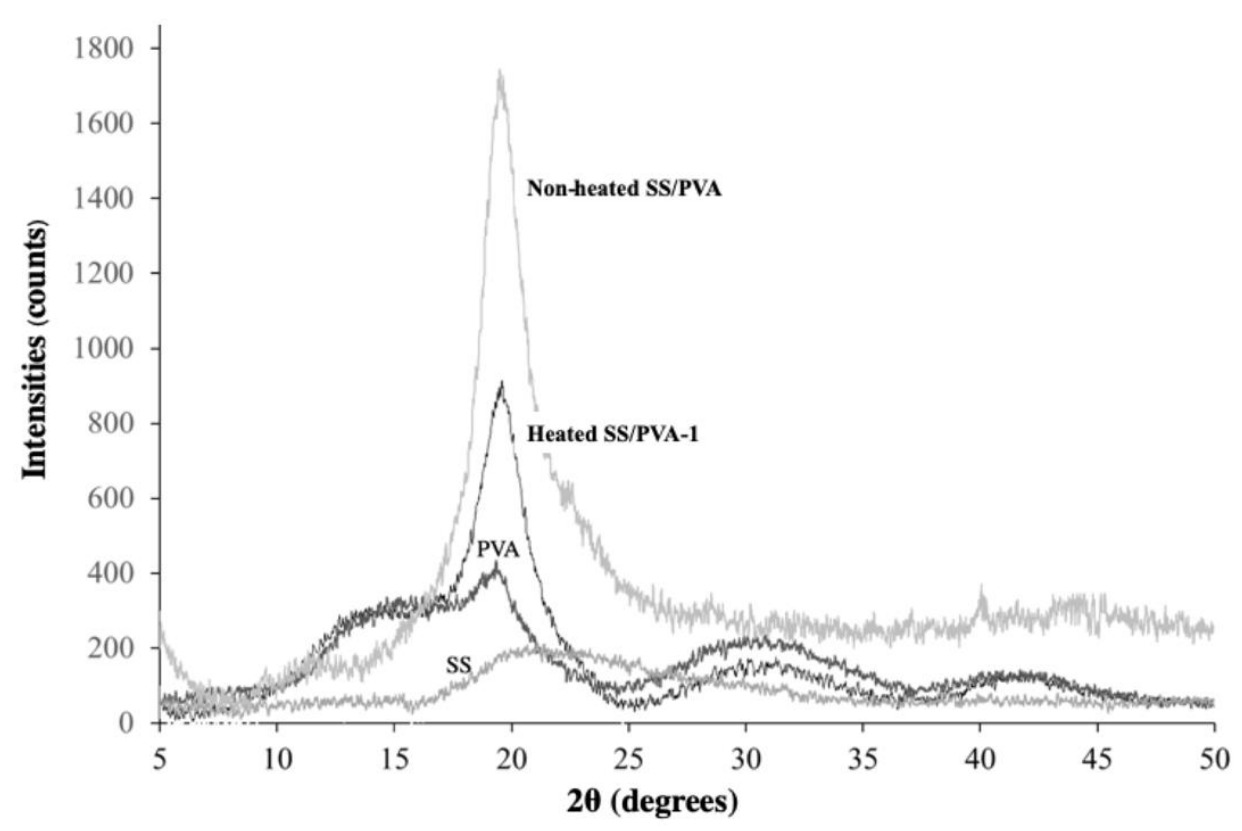

Figure 5. XRD patterns of SS, PVA, non-heated SS/PVA and heated SS/PVA-1 nanofibers.

In summary, the fabrication of environmentally friendly nanofibers was successfully 
established by combining SS with PVA and electrospinning from distilled water at elevated temperature. Temperature plays a key role in the SS sol-gel transition and thus influences the final formation of fibers, where heating the SS/PVA solution to $70^{\circ} \mathrm{C}$ produces mechanically stronger fibers with better morphology (less beads and micro-droplets), and higher hydrophobicity than fibers from non-heated SS/PVA solutions. Consequently, this method was selected for further study on the effect of SS and PVA concentration on the properties of the fibers.

\subsection{Heated SS/PVA electrospun nanofibers: Effect of SS and PVA concentration}

\section{Morphology}

The surface morphology of PVA at different concentrations and heated SS/PVA electrospun nanofibers (all samples listed in Table 2) was observed by SEM (see Fig. 6). Ultra-fine diameter fibers (60 $\mathrm{nm}$ diameter) with extensive bead formation was observed in $5 \%$ w/v PVA, while higher PVA concentrations produce thicker fibers with significantly less beads. All $5 \%$ w/v PVA incorporated with different loading of SS (EP1, EP2 and EP3) show fine fibers with beads, especially at $3 \mathrm{wt} \% \mathrm{SS}$ (EP3), which also produced micro-droplets. The formation of beads here is attributed to the low concentration of PVA and SS, which results in a low degree of chain entanglement $[36,49]$. Higher concentrations of PVA (7.5 and $10 \% \mathrm{w} / \mathrm{v})$ lead to more entanglements and thus enhanced viscoelastic properties, which results in better fiber formation with less beads. Addition of SS into 7.5 and $10 \%$ w/v PVA (EP4-EP9) causes the formation of beads, especially when a small amount of SS was added to $7.5 \%$ w/v PVA (EP4). This is again due to a low concentration of SS and/or its low molecular weight, which leads to a low degree of chain entanglement. Bigger diameter SS/PVA fibers were obtained when higher PVA and SS loadings were used. The fibrous mats of EP4-EP9 are soft and flexible and have the appearance of the fiber mat shown in Fig. 2. They also have sufficient mechanical strength to be peeled from the collector without tearing. From these morphology observations together with good fiber formation during electrospinning, samples of EP4-EP9 (especially EP5 and EP8) taken forward for further detailed investigation. 


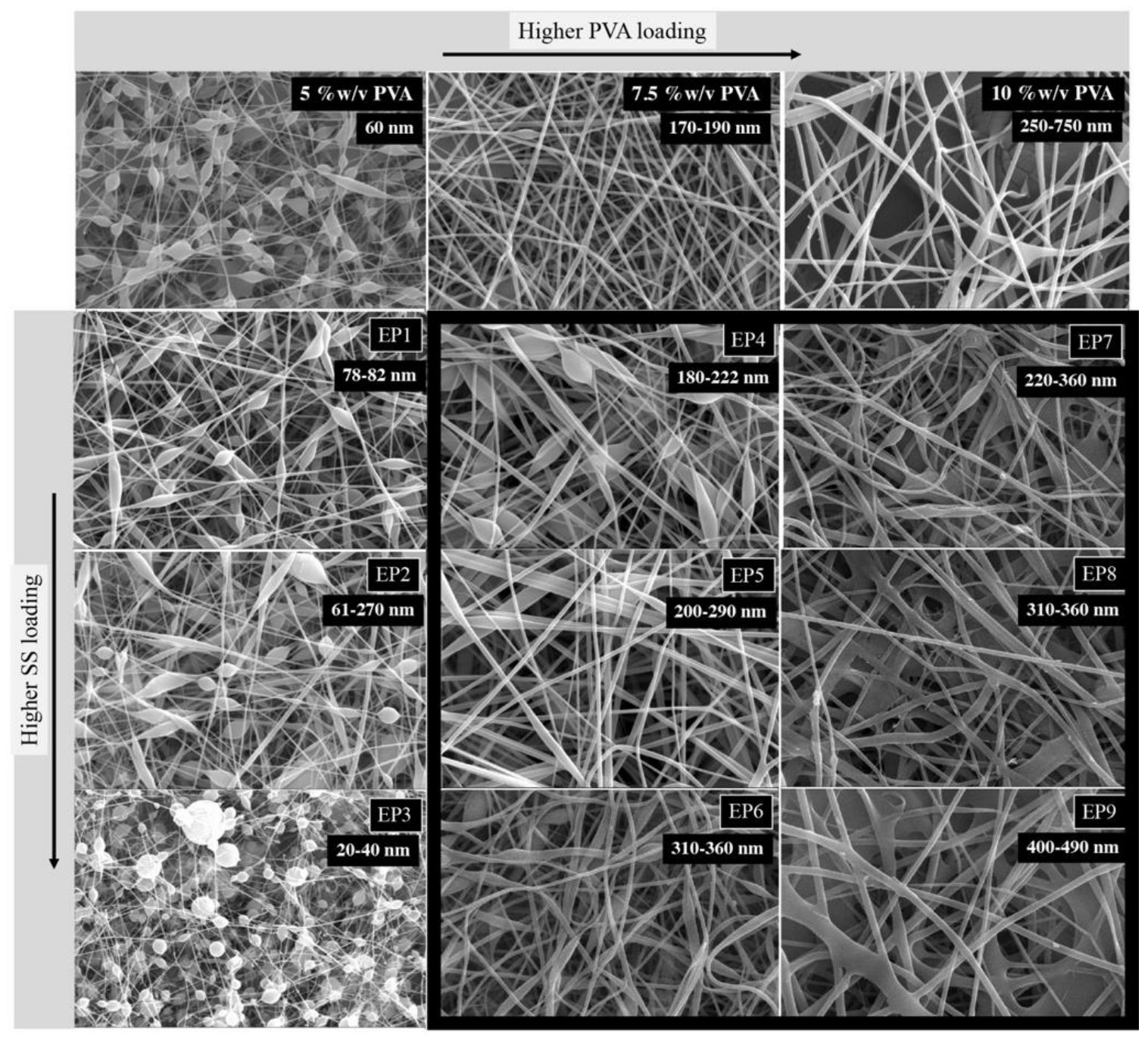

Figure 6. SEM images of PVA at different concentrations and heated SS/PVA nanofibers (EP1-9). Soft, flexible and strong fibers that can be readily peeled off the collector are shown in the black square (EP4-9).

\section{Intermolecular bonding}

Fig. 7 shows the FT-IR spectra of samples EP1-9. All samples show a small shift in amide I and amide II peaks to higher wavenumbers when compared to neat SS, while the broad bands (3700$3000 \mathrm{~cm}^{-1}$ ) of N-H stretching of SS and H-bonded -OH of PVA simply show the spectrum of the two homopolymers, which can be described as a miscible blend [50,51]. This miscible blend suggests that there are molecular interactions between PVA and SS. At higher SS concentrations, H-bonded -OH shows broader peaks and is shifted to a lower wavenumber due to the high degree of hydrogen bonding. However, the amide II (-NH bending) peak becomes broader and is shifted to higher wavenumber when using higher PVA contents due to increased molecular interactions between -OH 
groups of PVA and - $\mathrm{NH}_{2}$ groups of $\mathrm{SS}$.

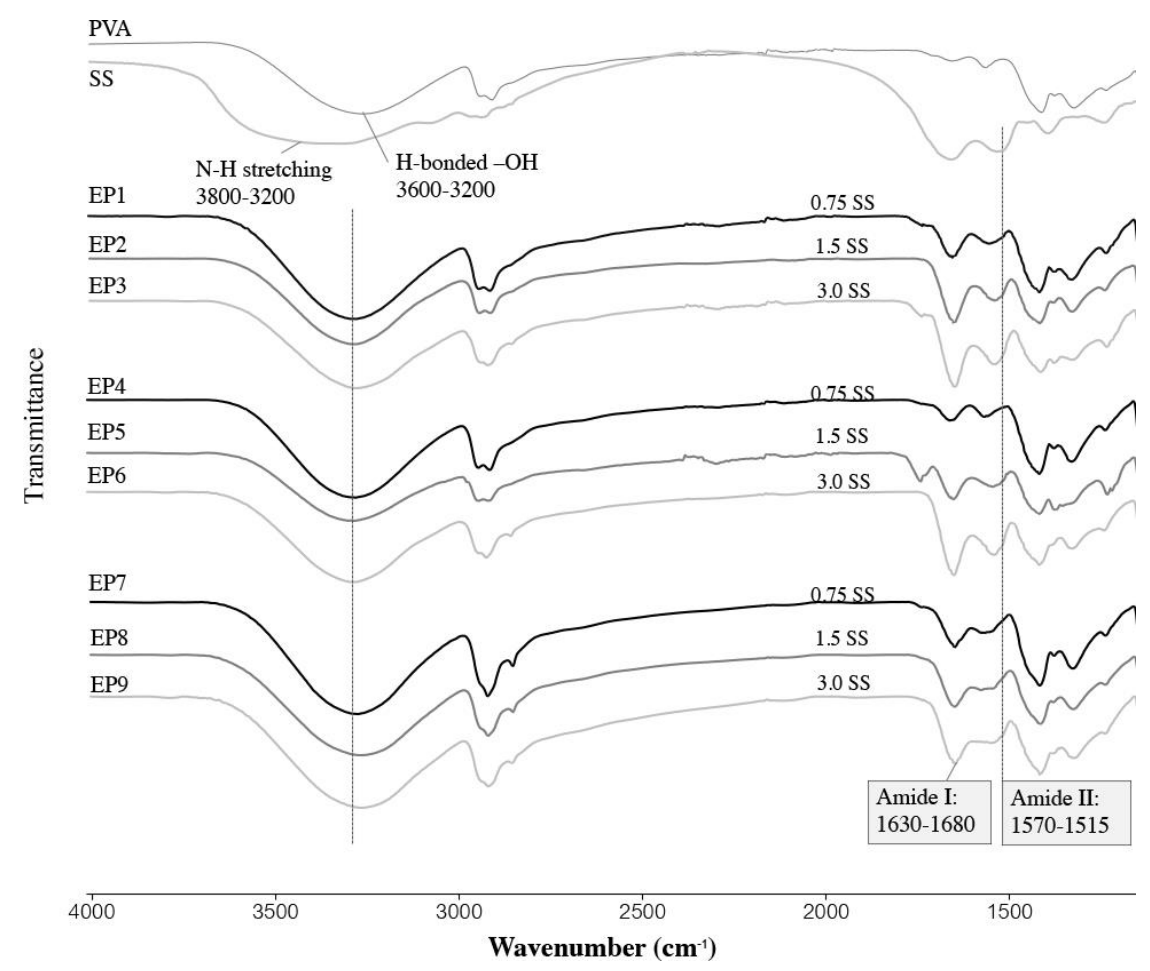

Figure 7. FT-IR spectra of SS, PVA and heated SS/PVA nanofibers with different concentrations of SS and PVA (EP1-9).

The effect of temperature on the molecular interactions between SS and PVA in the nanofibers of heated SS/PVA was also observed by FT-IR (heating samples from $27{ }^{\circ} \mathrm{C}$ to $70{ }^{\circ} \mathrm{C}$ ), as shown in Fig.8. All samples (EP4, EP6 and EP9 are provided here as representative exemplars) show that the bands from the H-bonded - $\mathrm{OH}$, amide I and amide II are shifted to higher wavenumbers when higher temperatures are applied, which shows increased interaction between PVA and SS. These results confirm that the electrospinning process of SS/PVA solutions should be undertaken above the gelation temperature (approximately $70^{\circ} \mathrm{C}$ ) of SS to maximize the molecular interactions between SS and PVA. 


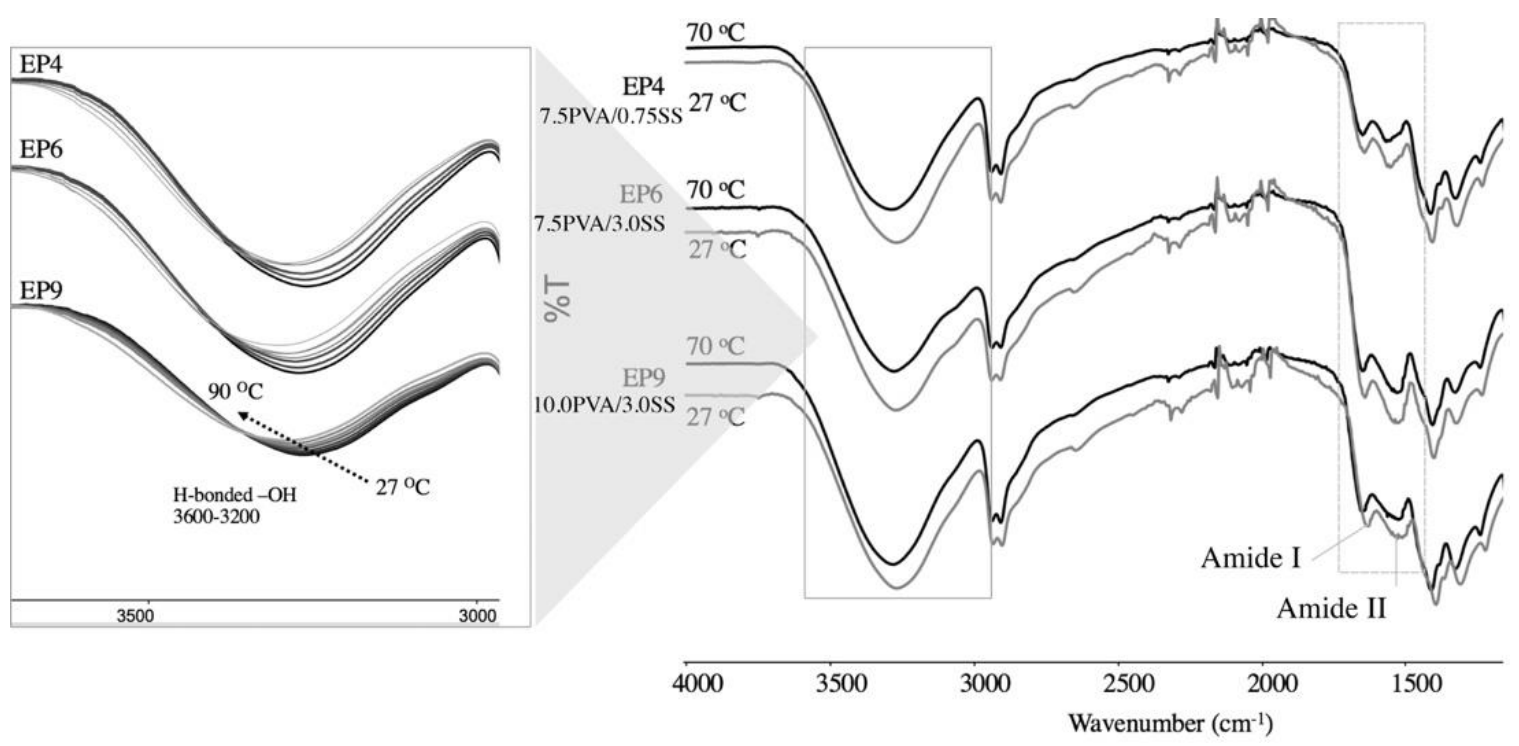

Figure 8. FT-IR spectra of representative heated SS/PVA nanofibers (EP4, EP6 and EP9) at 27

${ }^{\circ} \mathrm{C}$ and $70{ }^{\circ} \mathrm{C}$ and the FT-IR band of $\mathrm{H}$-bonded -OH testing from $27{ }^{\circ} \mathrm{C}$ to $90{ }^{\circ} \mathrm{C}$ (inset left).

\section{Crystallinity}

Fig. 9 shows the XRD patterns of all SS/PVA nanofibers on the same scale. The crystalline peaks of PVA are present at $\sim 19^{\circ}$ and their intensities were shown to be dependent on the concentration of PVA. In the set of EP1-3 (5 \%w/v PVA), different loadings of SS had only a small effect on the crystallinity of the nanofibers. In the set of EP7-9 (10\%w/v PVA), the disruption of the PVA crystal structure was observed when higher SS loadings were used. Here, the SS chains are able to mix with the PVA chains in solution and then form fibers with no beads after spinning (see Fig. 6), with higher SS loading more intermolecular interaction can occur. In the set of EP4-6 (7.5\%w/v PVA), however, the EP5 composition (mid SS concentration of $1.5 \% \mathrm{w} / \mathrm{w}$ ) shows the highest crystalline peak (similar to EP8). From these XRD results, it appears that SS is needed to induce PVA crystallinity, but too much SS loading suppresses the formation of PVA crystals. 


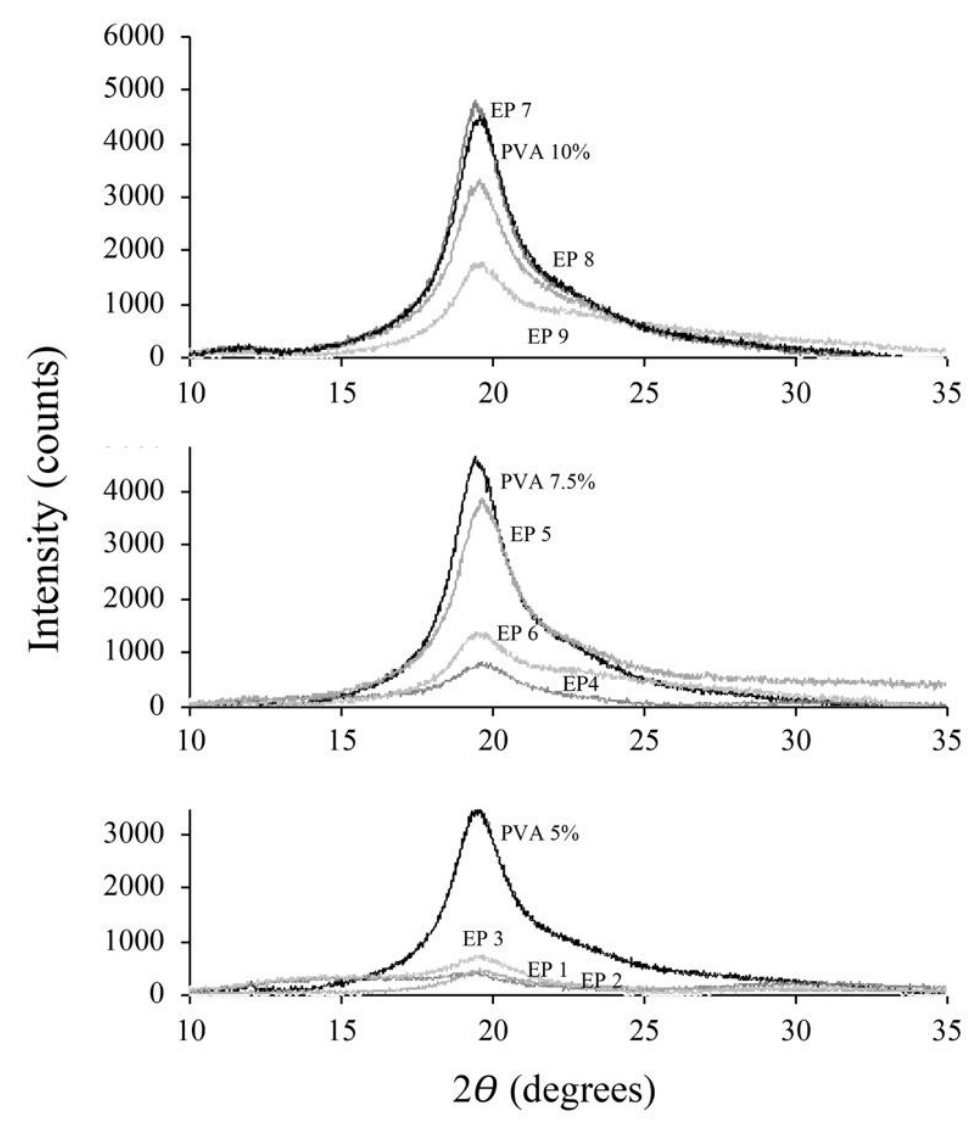

Figure 9. XRD patterns of heated SS/PVA nanofibers with different concentrations of SS and PVA.

\section{Degradation}

The degradation properties of scaffolds can be of particular importance with many scaffolds manufactured for the treatment of nonhealing wounds being conventionally designed to degrade [52]. In vitro degradation tests were carried out for 42 days on all PVA/SS nanofibers (made from different concentrations of PVA and SS), along with neat PVA and the results are shown in Fig. 10. The data show that all samples exhibited weight loss in range of 30-50\%. High SS loading appears to promote higher degradation rates in EP1-6. However, the weight loss of EP7-9 (at PVA high concentrations of $10 \% \mathrm{w} / \mathrm{v}$ ) are not significantly different. This is possibly due to the trapping of SS chains in the regions of high PVA contents. 

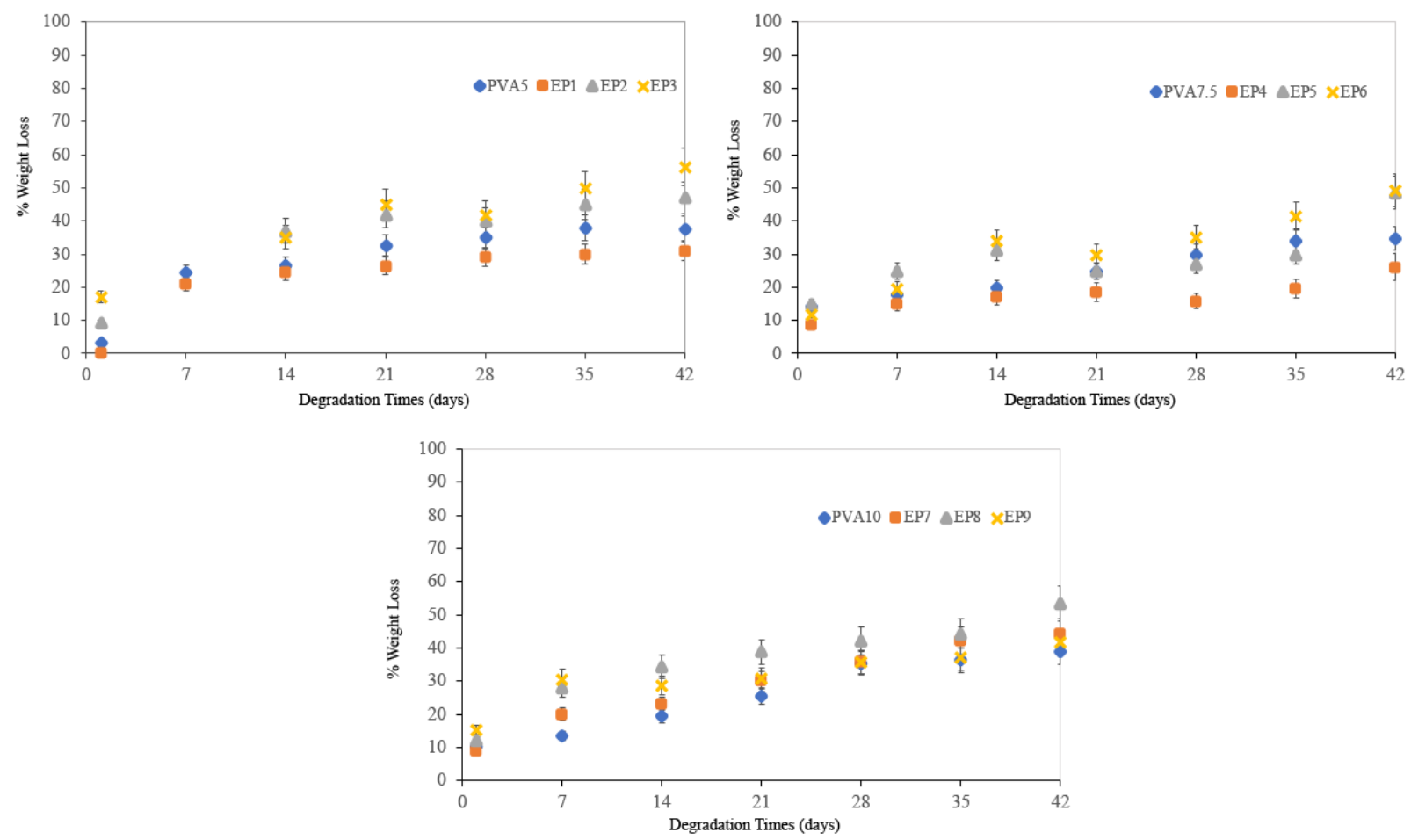

Figure 10. In vitro degradation of heated SS/PVA nanofibers with different concentrations of SS and PVA.

\section{In vitro cytotoxicity}

The in vitro cytotoxicity tests of SS/PVA nanofibers were examined using XTT assays toward NHDF cell line and the results are shown in Fig. 11. There is no difference in cell viabilities (for 24 hours) of all the control and test samples (EP5: 1.5SS/7.5PVA and EP8: 1.5SS/10PVA) and they were over $98 \%$. In addition, the optical microscopic images of the cells show that the morphology of the cells on our samples is the same as those on the control. The cell viability study suggested that the electrospun SS/PVA nanofibers are found to be non-toxic materials with good biocompatibility. 

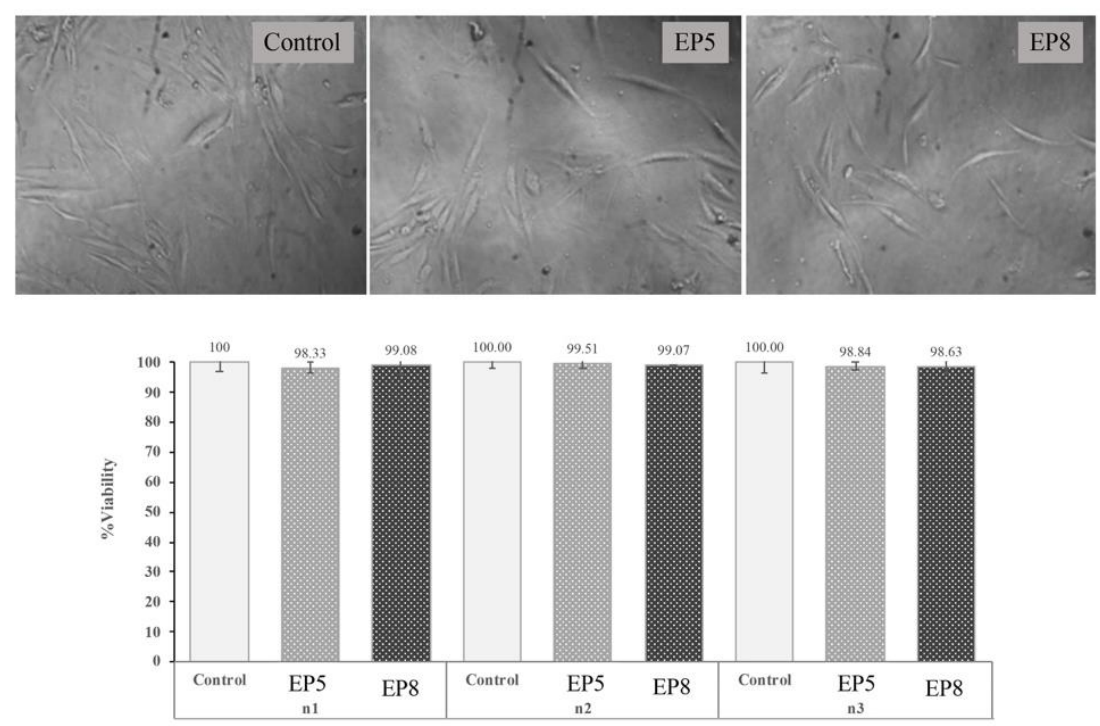

Figure 11. In vitro cytotoxicity of NHDF cell line on SS/PVA nanofibers together with the optical microscopic images of the cells on fibers. All experiments were performed three times with three replicate wells for every sample and control per assay.

\section{Conclusions}

Water stable, but biodegradable SS/PVA nanofibrous fabrics have been successfully fabricated from distilled water, without the use of crosslinking agents via an environmentally friendly route. By heating the electrospinning solution to $70{ }^{\circ} \mathrm{C}, \mathrm{SS}$ (in distilled water) was found to be a sol (random coil structure), whereas it is a gel ( $\beta$-sheet structure) at room temperature. The random coil structure in the sol enhanced molecular interaction between PVA and SS and promoted the fabrication of nanofibers with better morphology and more hydrophobic character (both PVA and SS are hydrophilic). PVA and SS loadings have been shown to effect the nanofiber diameter, formation of beads, crystallinity, molecular bonding and degradation of the resultant nanofibers. All fibers at 7.5 and $10 \%$ w/v PVA (EP4-9, especially EP5 and EP8) showed good physical properties, such as ease of fabrication and simple removal from the collector, owing to their enhanced mechanical strength. These fibers were also shown to be non-toxic to fibroblast cells. With their enhanced properties; such as non-toxicity, good water stability, biodegradability and good physical and mechanical properties, 
such PVA/SS nanofiber mats have the potential for use as skin tissue regenerative scaffolds. Crucially, these bio-inspired materials containing SS can be fabricated from an environmentally friendly process.

\section{Acknowledgements}

This work was supported by the Annual Government Statement of Expenditure (2018) of Thailand [grant number 658639]. Also thank to the Science Lab Centre, Faculty of Science, Naresuan University for supporting XRD, DSC, CA, SEM and FTIR measurements. Special thanks must also go to the Clinical Microbiology Laboratory, Department of Medical Technology, Faculty of Allied Health Sciences, Naresuan University.

\section{References}

[1] Venkatesan J, Lowe B, Pallela R and Kim S-K, Chitosan-based polysaccharide biomaterials, in: Polysaccharides: Bioactivity and Biotechnology, by Ramawat KG and Mcrillon J-M. Springer, Switzerland, pp. 1837-1850 (2015).

[2] Ampawong S and Aramwit P, J Bioact Compat Polym 32(6): 582-595 (2017).

[3] Handipour-Goudarzi E, Montazer M, Latifi M and Aghaji AAG, Carbohydr Polym 113: 231-239 (2014).

[4] Zhao R, Li X, Sun B, Zhang Y, Zhang D, Tang Z, Chen X and Wang C, Int J Biol Macromol 68: 92-97 (2014).

[5] Mi FL, Tan YC, Liang HF and Sung HW, Biomaterials 23: 181-191 (2002).

[6] Sugimoto M, Morimoto M, Sashiwa H, Saimoto H and Shigemasa Y, Carbohydr Polym 36: 49-59 (1998).

[7] Chen JP, Chang GY and Chen JK, Colloids Surf, A: Physicochem Eng Aspects 313: 183$188(2008)$.

[8] Dhandayuthapani B, Krishnan UM and Sethuraman S, J Biomed Mater Res Part B: Appl Biomater 94: 264-272 (2010). 
[9] Hu WW and Yu HN, Carbohydr Polym 95: 716-727 (2013).

[10] Cai ZX, Mo XM, Zhang KH, Fan LP, Yin AL, He CL and Wang HS, Int J Mol Sci 11: 3529-3539 (2010).

[11] Yan S, Li X, Dai J, Wang Y, Wang B, Lu Y, Shi J, Huang P, Gong J and Yao Y, Mater Sci Eng, C 79: 436-444 (2017).

[12] Das P, Ojah N, Kandimalla R, Mohan K, Gogoi D, Dolui SK and Choudhury AJ, Int J Biol Macromol 114: 1026-1032 (2018).

[13] Yooyod M, Ross GM, Limpeanchob N, Suphrom N, Mahasaranon S and Ross S, Eur Polym J 81: 43-52 (2016).

[14] Ross S, Yooyod M, Limpeanchob N, Mahasaranon S, Suphrom S and Ross GM, Exp Polym Polymer Lett 11(9): 719-730 (2017).

[15] Kunz RI, Branscalhao RMC, Ribeiro LFC and Natali MRM, BioMed Res Int 2016: 19 pages (2016).

[16] Zhang X, Khan Md MR, Yamanoto T, Tsukada M and Morikawa H, Int J Biol Macromol 50: $337-347$ (2012).

[17] Yang C, Xue R and Zhang Q et al, Mater Sci Eng, C 81: 303-313 (2017).

[18] Gilotra S, Chouhan D, Bhardwaj N, Nandi SK and Mandal BB, Mater Sci Eng, C 90: 420$432(2018)$.

[19] He M, Hu H and Wang P et al, Int J Biol Macromol 117: 323-330 (2018).

[20] Sapru S, Das S, Mandal M, Ghosh A and Kundu SC, Acta Biomaterialia 78: 137-150 (2018).

[21] Chao S, Li Y, Zhao R, Zhang L, Li Y, Wang C and Li X, J Drug Delivery Sci Techno 44: 440-447 (2018) .

[22] Park CJ, Ryoo J, Ki CS, Kim JW, Kim IS, Gae DG and Um IC, Int J Biol Macromol 119: 821-832 (2018). 
[23] Ang-atikarnkul P, Watthanophanit A and Rujiravanit R, Compos Sci Technol 96: 88-96 (2014).

[24] Kundu B and Kundu SC, Biomaterials 33: 7456-7467 (2012).

[25] Rebia RA, Rozet S, Tamada Y and Tanaka T, Polym Degrad Stab 154: 124-136 (2018).

[26] Li R, Lin J, Fang YY and Huang Y et al, Ceram Int 44: 22439-22444 (2018).

[27] He H, Cai R, Wang Y and Tao G et al, Int J Biol Macromol 104: 457-464 (2017).

[28] Park CJ, Ryoo J, Ki C, Kim JW, Kim IS, Bae DG and Um IC, Int J Biol Macromol 119: 821-832 (2018).

[29] Koosh M and Mirzadeh H, J Biomed Mater Res, A 103(9): 3081-3093 (2015).

[30] Agarwal S, Greiner A and Wendorff JH, Prog Polym Sci 38: 963-991 (2013).

[31] Wang L, Topham PD, Mykhaylyk OO, Howse JR, Bras W, Jones RAL and Ryan AJ, Adv Mater 19: 3544-3548 (2007).

[32] Xue J, Xie J, Liu W and Xia Y, Acc Chem Res 50: 1976-1987 (2017).

[33] Wang L, Topham PD, Mykhaylyk OO, Yu H, Ryan AJ, Fairclough JPA and Bras W, Macromol Rapid Commun 36: 1437-1443 (2015).

[34] Wu J, Li X, Wu Y, Liao G, Johnston P, Topham PD and Wang L, Appl Surf Sci 422: 769777 (2017).

[35] Abudula T, Saeed U, Salah N, Memic A and Al-Turaif H, J Nanosci Nanotechnol 18: 8240-8251 (2018).

[36] Tuancharoensri N, Ross GM, Mahasaranon S, Topham PD and Ross S, Polym Int 66(11): 1463-1472 (2017)

[37] Nune M, Krishnan UM and Sethuraman S, Mater Sci Eng, C 62: 329-337 (2016).

[38] Liu M, Duan X-P, Li Y-M, Yang D-P, Long Y-Ze, Mater Sci Eng, C. 76: 1413-1423 (2017).

[39] Zong XH, Kim K, Fang DF, Ran SF, Hsiao BS and Chu B, Polymer 43: 4403-4412 (2002) 
[40] Freddi G, Tsukada M and Beretta S, J Appl Polym Sci 71: 1563-1571 (1999).

[41] Padamwar MN and Pawar AP, J Sci Ind Res 63: 323-329 (2004).

[42] Andreas B and Zscherp C, Q. Rev. Biophys. 35: 369-430 (2002).

[43] Fraser RBD and MacRae TP, Conformation of Fibrous Proteins and Related Systhetic Polypeptides, in Chapter 13. Silks, by Fraser R. Academic Press, New York, pp. 293-343 (1973).

[44] Takasu Y and Yamada H et al, Biosci Biotechnol Biochem 66: 2715-2718 (2002).

[45] Jo YN, Park B and Um IC, Int J Biol Macromol 81: 936-941 (2015).

[46] Jang MJ and I.C. Um IC, Eur Polym J 93: 761-774 (2017).

[47] Kweon HY, Yeo JH, Lee KG, Lee YW, Park YH, Nahm J and Cho C, Macromol Rapid Commun 21: 1302-1305 (2000).

[48] Zhu LJ, Arai M and Hirabayashi K, J Seric Sci Jpn 65(4): 270-274 (1996).

[49] Yu JH, Fridrikh SV and Rutledge GC, Polymer 47: 4789 - 4797 (2006).

[50] Ross S, Mahasaranon S and Ross GM, J of Appl Polym Sci 132(14): 41780(1-8) (2015).

[51] Koenig JL, In Spectroscopy of Polymers, by Koenig JL, Elsevier, New York, pp. 35 (1999).

[52] Turner NJ and Badylak SF, Adv Wound Care 4(8): 490-500 (2015). 